\title{
3D Full-field Transmission X-ray Microscopy Based on Equally Sloped Tomography at Shanghai Synchrotron Radiation Facility
}

\author{
$\underline{\text { Yuqi Ren }}^{1}$, Yudan Wang ${ }^{1}$, Guangzhao Zhou ${ }^{1}$, Guohao Du ${ }^{1}$, Biao Deng ${ }^{1,2,{ }^{*}}$, Honglan Xie ${ }^{1,2}$ and Tiqiao \\ $\mathrm{Xiao}^{1,2}$. \\ 1. Shanghai Institute of Applied Physics, Chinese Academy of Sciences, 239 Zhangheng Road, Shanghai, \\ 201204, China. \\ 2. University of Chinese Academy of Sciences, No. 19A Yuquanlu, Beijing, 100049, China. \\ * Corresponding author, email_dengbiao@sinap.ac.cn
}

Three-dimensional full-field transmission X-ray microscopy (TXM) is capable of 3D imaging of morphology and structures with spatial resolution down to a few tens of nanometers. Many of hard Xray TXM facilities, both based on synchrotron radiation and tabletop X-ray sources, has been built up all over the world [1-3]. Recent developments have demonstrated that hard X-ray TXM is an effective and unique technique for non-destructive investigation of battery material under in-situ environment [2, 3]. Not only morphology of 2D and 3D structures, but also elemental distribution and chemical states of battery material can be obtained simultaneously.

At present, the BL13W1 is the only hard X-ray imaging beamline at Shanghai Synchrotron Radiation Facility (SSRF), which aims to investigate the 3D microstructure of biomedical samples and low element-materials with micrometer resolution. In order to promote the nano imaging ability at SSRF, a hard TXM system has been designed and installed at BL13W1, as shown in Figure 1. A condenser is used to illuminate X-ray beam onto the sample. The condenser integrated with a central stop could provide a hollow illumination.

A zone plate-type beamshaper (Fabricated by Paul Scherrer Institute, Switzerland), located at 33m downstream from the source, was chosen as the condenser. It has the outermost zone width of 70nm, gold structures of $1.1 \mu \mathrm{m}$ in height. The resulting aspect-ratio of 15 ensures the diffraction efficiency up to $8 \%$ at $10 \mathrm{keV}$. The beamshaper collects $1.8 \times 1.8 \mathrm{~mm}^{2}$ of the X-ray beam and produces an uniform illumination of $50 \times 50 \mu \mathrm{m}^{2}$ onto the sample at a distance of $\mathrm{f}_{\text {condenser }}=1459 \mathrm{~mm}$ downstream. A Fresnel zone plate (FZP) was employed as an objective lens. It has a focal length of $\mathrm{f}_{\mathrm{FZP}}=56.5 \mathrm{~mm}$ at $10 \mathrm{keV}$, an outermost zone width of $70 \mathrm{~nm}$, a zone height of $1 \mu \mathrm{m}$, and a diameter of $100 \mu \mathrm{m}$. The Hamamatsu Flash $\mathrm{X}$-ray CCD with the effective pixel size of $6.5 \mu \mathrm{m}$ is adpoted as the detector. The distance between the zone plate and sample is a little longer than $\mathrm{f}_{\mathrm{FZP}}$. The distance between FZP and detector ranges from $6500 \mathrm{~mm}$ to $7000 \mathrm{~mm}$, which could result in a magnification of $115-124$. The numerical aperture of the illumination from beamshaper should match with that of the objective lens in order to obtain optimum resolution [4]. A 6m-long pipeline filled with Helium gas was placed between FZP and detector to reduce the effect of the X-ray attenuation in the air. Since there is no focusing optics in the BL13W1, it leads to a lower flux density at the sample and results in a considerable longer exposure time, typically one minute or more per image.

A set of limited equal-angle 2D TXM projections (typical several hundreds of projections over 180 degrees) were used to reconstruct the 3D structural information by conventional filtered back projection (FBP) algorithm. This means a very long data-collection time by the proposed system. In order to reduce the total acquisition time, a novel data acquisition and reconstruction method named as equally-sloped 
tomography (EST), has been introduced in TXM. It is demonstrated that EST could cut the projections up to 75\% while maintain the same image quality compared with conventional CT method [5].

To evaluate the spatial resolution of the full-field TXM system, an X-ray Chart with radial patterns (from NTT Advanced Technology Corporation) is used. The detector is placed at 6900mm downstream the FZP resulting in a magnification factor of 122. Effective pixel size of 53nm can be achieved. Flatfield and dark-field images were acquired for correction of illumination and CCD artefacts. As shown in Figure 2, the innermost stripes down to $100 \mathrm{~nm}$ can be distinguished, which means spatial resolution of $100 \mathrm{~nm}$ has been realized. The effective FOV is about $47 \mu \mathrm{m} \times 47 \mu \mathrm{m}$. 3D investigation of tantalum powder (with the average diameter of $1 \mu \mathrm{m}$ ) was carried out on the EST-based TXM system. The alignment of the rotation center of all projections was performed using cross-correlations method. As shown in Figure 3, the tantalum powder-formed clusters can be visualized clearly.

The hard X-ray TXM system was successfully self-designed and self-constructed at SSRF. The system is capable of 2D imaging of 100nm resolution. By the aid of EST technique, it provides 3D tomographic capabilities for characterization of materials with much shorter collection time. EST-based TXM will be constructed in the dedicated 3D hard X-ray nano imaging beamline proposed in SSRF phase II project.

References:

[1] Q. Yuan et al, J. Synchrotron Radiat. 19 (2012) 1021.

[2] J. Wang et al, Nat. Commun. 6 (2015) 7496.

[3] F. Yang et al, Nano Lett. 14 (2014) 4334.

[4] B. Feng et al, Chin. Opt. Lett. 14 (2016) 84.

[5] Y. Ren\#, Y. Wang" et al, J. X-ray Sci. Technol. 24 (2016) 79.

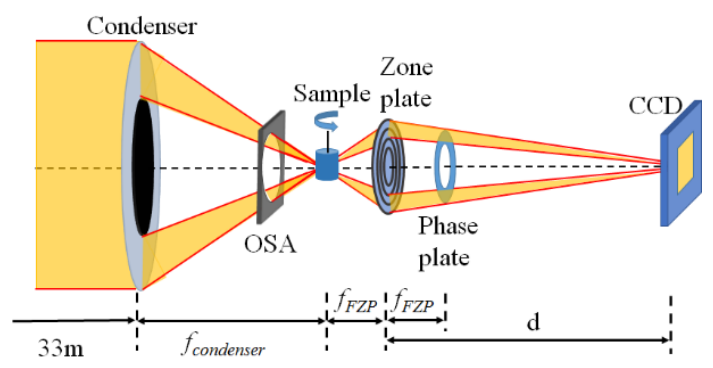

Figure 1 Optical layout of full field TXM system.
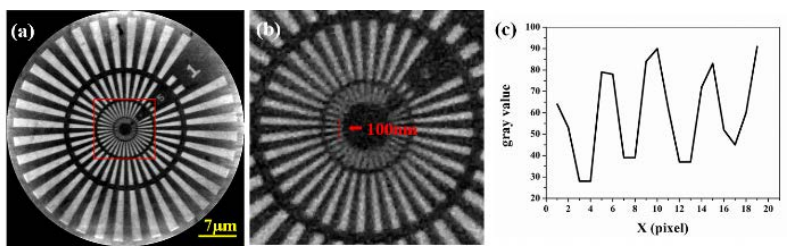

Figure 2 (a) Image of the Siemens star by nano-CT system, (b) The amplified image of the part indicated by frame in (a), (c) The line profile of solid line in (b).
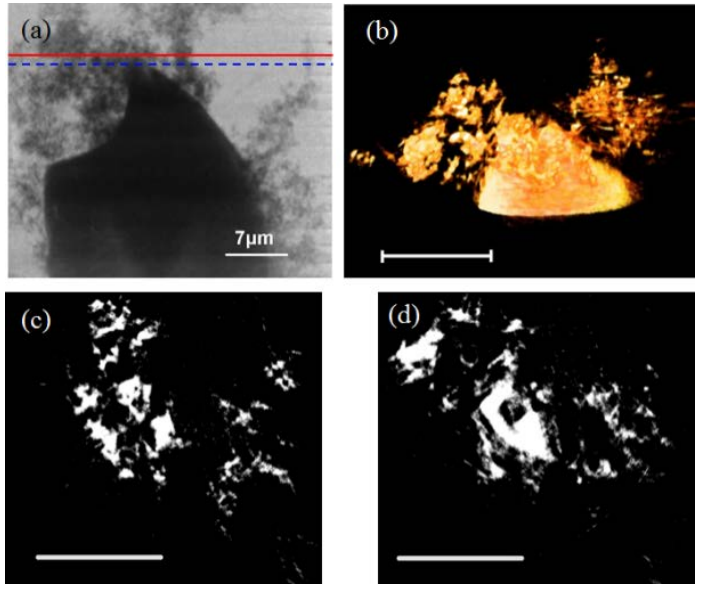

Figure 3 Results of EST-based TXM. (a) projection, (c) and (d) are slices reconstructed by EST for lines 1 and 2 in (a) using 64 EST projections, (b) 3D rendering of top part of the sample. Scale bar: $7 \mu \mathrm{m}$. 